\title{
In vitro assessment of an engineered tBID-based safety switch system in human $\mathrm{T}$ lymphocytes
}

\author{
Jiamiao Lu” ${ }^{\#}$ Patrick Collins”, Ki Jeong Lee, Chi-Ming Li^, Songli Wang \\ Genome Analysis Unit, Amgen Global Research, South San Francisco, CA, USA \\ Contributions: (I) Conception and design: J Lu, P Collins, S Wang; (II) Administrative support: CM Li, S Wang; (III) Provision of study materials \\ or patients: J Lu, P Collins, KJ Lee; (IV) Collection and assembly of data: J Lu; (V) Data analysis and interpretation: All authors; (VI) Manuscript \\ writing: All authors; (VII) Final approval of manuscript: All authors. \\ \#These authors contributed equally to this work. \\ Correspondence to: Chi-Ming Li, PhD, MSc; Songli Wang, PhD. 1120 Veteran Blvd, ASF1-2, South San Francisco, CA 94080, USA. \\ Email: chimingl@amgen.com; songliw@amgen.com.
}

Background: Cell therapy as a promising therapeutic modality to treat cancer has been intensively studied for decades. However, the clinical trials have indicated that patients under $T$ cell therapy may develop severe cytokine release syndrome resulting in hospitalization or even death. Furthermore, genetic modifications to promote proliferation and persistence of $\mathrm{T}$ cells could result in high numbers of long-lived engineered cells in patients after treatment.

Methods: We incorporated the pro-apoptotic truncated BH3 interacting-domain death agonist (tBID) with the mutant ecDHFR destabilizing domain to form a novel recombinant protein as the major component of an engineered tBID-based safety switch system, which would be unstable and quickly degraded in the absence of trimethoprim (TMP) but, upon TMP treatment, should become stabilized and allow tBID to induce cell death experimentally.

Results: The novel tBID-based safety switch could be regulated through a small molecule inducer, TMP, to control undesired toxicity or ablate the engineered cells as needed. We systematically compared and assessed several tBID-based safety switch constructs with the clinically validated safety switches, including human herpes simplex virus thymidine kinase (HSV-TK) and inducible Caspase 9 (iCasp9). With optimization, we were able to achieve significant killing potency in vitro in Jurkat or human primary $\mathrm{T}$ cells.

Conclusions: We demonstrated that our engineered tBID-based safety switch was able to eliminate up to $\sim 90 \%$ of transduced human primary $\mathrm{T}$ cells within $72 \mathrm{~h}$ after activation, providing an alternative switch system to manage safety concerns for cell therapy.

Keywords: Truncated BID (tBID); safety switch; T lymphocyte; cell therapy

Submitted Jan 28, 2021. Accepted for publication Apr 25, 2021.

doi: $10.21037 /$ atm-21-443

View this article at: https://dx.doi.org/10.21037/atm-21-443

\section{Introduction}

Over the last few decades cell therapies have opened opportunities to new treatments for cancers, autoimmune disorders, and inherited diseases. These treatments often involve transplantation of genetically-engineered stem cells or specific cell types of interest. However, the potentials of the exogenously infused cells to malignant transformation and the toxicities of the transgene-expression products have led to the emergence of safety concerns. Numerous safety switch systems have been incorporated and developed to

$\wedge$ ORCID: 0000-0002-2740-5652. 
mitigate undesired toxicities in the donor cells, resulting in selective depletion when severe toxicities, for examples cytokine release syndromes, arise.

A safety switch derived from the human herpes simplex virus thymidine kinase type 1 gene, HSV-TK, has been clinically validated in allogenic donor $\mathrm{T}$ cell infusion to treat tumor and Epstein Barr virus (EBV) lymphoproliferative disease after hemopoietic stem cell transplantation (1-3). The killing activity of HSV-TK is activated by an antiviral prodrug, ganciclovir (GCV), which hinders the administration of GCV for cytomegalovirus infections. In recent years a robust fast killing safety switch system, inducible Caspase 9 (iCasp9), has emerged $(4,5)$. In this system, the human Caspase 9 protein lacking the caspase activation and recruitment (CARD) domain is fused to a human FK506-binding protein (FKBP) derived drug-binding domain. Without activation the Caspase 9 fusion protein stays in monomer form. By binding with a chemical induction of dimerization (CID) drug, AP1903, the Caspase 9 fusion protein dimerizes and in turn activates the cellular apoptosis events. The feasibility of iCasp9 has been tested clinically in the patients who received haploidentical hematopoietic stem cell transplantation, in which the genetically-engineered donor $\mathrm{T}$ cells decreased by more than $90 \%$ within 30 minutes after CID infusion (5). The efficacy of iCasp9 in controlling serious adverse events caused by genetically-engineered chimeric antigen receptor (CAR) T-cell therapy was also validated in phase 1 clinical trials (6). However, while iCasp9 responded quickly in 30 minutes upon induction, it could take a few days for the HSV-TK system to show effects.

As one of pro-apoptotic BCL-2 family members, BH3 interacting-domain death agonist (BID) functions as a $\mathrm{BH} 3$ domain only death agonist (7). The cytosol-localized fulllength BID is a substrate of Caspase 8 (8). Cleavage of BID by Caspase 8 releases its pro-apoptotic activity. The truncated BID (tBID) then translocates from cytosol to mitochondria where it induces clustering of mitochondria around nucleus, cytochrome c release, and then loss of mitochondrial membrane potential, leading to cell shrinkage and nuclear condensation (8).

While tBID demonstrates many of the properties of an ideal safety switch, expression of the protein itself would lead to cell death and so it needs to be coupled to some sort of a molecular switch. The ideal switch would result in no background expression of tBID until induced by a small molecule. Ideally, the inducer would be commercially available, clinically validated, inexpensive, well-characterized compound that can pass through both the blood-brain barrier (BBB) and the blood-retinal barrier (BRB). Trimethoprim (TMP) is a frequently used antibiotic that binds to bacterial dihydrofolate reductase (DHFR) with $1,000 \times$ selectivity compared to human DHFR. Multiple E. coli DHFR (ecDHFR) destabilizing domain mutants have been engineered for construction of the destabilizing domains (9). By fusing such a destabilizing domain to the other domain or protein of interest, the entire fusion protein should be unstable and rapidly degraded. Addition of TMP stabilizes the destabilizing domain in a rapid, reversible and dose-dependent manner $(10,11)$, suggesting that cell death mediated by TMP administration may be a practical method to manage or attenuate safety concerns in cell therapy.

In this study, we took the advantage of the ecDHFR destabilizing domain system fused with tBID to develop an alternative safety switch system that can not only gradually but also thoroughly deplete engineered donor cells to expand the options of safety switch systems. The safety switch candidates were constructed by fusing tBID with the mutants of the ecDHFR destabilizing domain at either $\mathrm{N}$ - or C-termini. The potencies of these tBID based safety switches were validated and assessed in vitro with or without TMP in both human Jurkat and primary pan-T cells.

We present the following article in accordance with the MDAR reporting checklist (available at http://dx.doi. org/10.21037/atm-21-443).

\section{Methods}

\section{Vector construction and cloning}

The iCasp9, NDHFR-tBID, HSV-TK, and tBIDCDHFR fragments were synthesized by GENEWIZ. These fragments were then cloned into in house lentiviral vectors. The M97A/D98A mutations were introduced into NDHFR-tBID by using In-Fusion site directed mutagenesis kit with forward primer 5'CCAGGTCGGGG ACAGCGCGGCGCGTAGCATCCCTCCGGGC3' and reverse primer 5'GCCCGGAGGGATGCTACGCGCCG CGCTGTCCCCGACCTGG3'.

\section{Cell culture and transduction}

The Jurkat cells (ATCC TIB-152) were cultured with RPMI1640 medium supplemented with $10 \%$ fetal bovine serum (FBS) and $100 \mu \mathrm{g} / \mathrm{mL}$ streptomycin. On the 
day performing transduction, lentiviral vector at desired multiplicity of infection (MOI) was added to $1 \mathrm{~mL}$ of $2 \times \mathrm{E}+05$ cells $/ \mathrm{mL}$ in $15 \mathrm{~mL}$ Falcon tube in PRMI1640 medium (Sigma-Aldrich, Cat\# R7388-500ML) with 10\% FBS and $5 \mu \mathrm{g} / \mathrm{mL}$ polybrene (Sigma-Aldrich, Cat\# TR-1003) then followed by gentle agitation. Spinoculation was performed by centrifugation of the tubes at $1,200 \times \mathrm{g}$ for $2 \mathrm{~h}$ at $32{ }^{\circ} \mathrm{C}$. Supernatant was aspirated off, resuspended cells with $2 \mathrm{~mL}$ PRMI1640 medium with $10 \%$ FBS, placed the cells in 12well plate and incubated the plate in incubator at $37^{\circ} \mathrm{C}$.

Human T-cell samples were purchased anonymously from the vendor and considered exempt from IRB approval. The frozen human pan-T cells (AllCells, LLC., Cat\# S-ISO004-06) were thawed and cultured with X-vivo 15 medium (Lonza, Cat\# BE02-053Q), 5\% heat-inactivated human serum (Sigma-Aldrich, Cat\# H3667-20ML), $1 \times$ Pen/Strep (Sigma-Aldrich, Cat\# P4333-100ML), human T-Activator CD3/CD28 Dynabeads (Gibco, Cat\# 111.31D) (at $1: 1$ cells: beads ratio) at $1 \times \mathrm{E}+06$ cells $/ \mathrm{mL}$ per well in 24-well plate on day 0. Performed CD3 staining on day 0 to confirm T cell purity. Transduction was performed on day 3 . First added fresh medium with IL-2 $100 \mathrm{U} / \mathrm{mL}$ to make the cell concentration at $0.5 \times \mathrm{E}+06$ cells $/ \mathrm{mL}$. Place $1 \mathrm{~mL}$ of cell suspension into each $15 \mathrm{~mL}$ falcon tube. Then added lentivirus at desired MOI and $8 \mu \mathrm{g} / \mathrm{mL}$ polybrene. Span the tubes at $1,800 \mathrm{rpm}(1,000 \mathrm{~g})$ for $45 \mathrm{~min}$ at $32{ }^{\circ} \mathrm{C}$. Finally removed lentivirus, replaced the medium at $0.5 \times \mathrm{E}+06$ cells $/ \mathrm{mL}$ with IL-2 $100 \mathrm{U} / \mathrm{mL}$, and culture cells at $37{ }^{\circ} \mathrm{C}$ tissue culture incubator. Changed medium when needed.

\section{Protein L magnetic bead purification of lentivirus transduced buman pan-T cells}

$1 \times \mathrm{E}+06$ transduced human pan-T cells were counted and washed with cold PBS on day 3 post lentivirus transduction. The cells were then resuspended in $1 \mathrm{~mL}$ cold PBS with $0.5 \%$ BSA and $0.05 \%$ Tween-20; $100 \mu \mathrm{L}$ of Protein L magnetic beads (Pierce Protein L Magnetic Beads, Cat\# 88850 ) were first washed with $1 \mathrm{~mL}$ cold PBS with $0.5 \%$ BSA and $0.05 \%$ Tween- 20 by gentle vortexing for $1 \mathrm{~min}$. Then collected the beads by using magnetic stand and removed supernatant. Then added the resuspended transduced human pan- $T$ cells into the tube with washed beads. Incubate at $4{ }^{\circ} \mathrm{C}$ with mixing for $1-2 \mathrm{~h}$. Collected enriched cells by magnetic stand and removed supernatant. Wash the cells with $1 \mathrm{~mL}$ cold PBS with $0.5 \%$ BSA and $0.05 \%$ Tween-20 twice by using magnetic stand. Removed the wash buffer after the final wash. Resuspended the final bead-cell pellet with fresh $\mathrm{X}$ vivo 15 with IL-2 $100 \mathrm{U} / \mathrm{mL}$ and incubated in cell culture flask at $37^{\circ} \mathrm{C}$. Beads fell off after incubation and could be removed by magnetic stand before next analysis.

\section{Flow cytometry and bead-based cell counting}

On day 3 post lentivirus transduction in human pan-T cells and Jurkat cells, prepared cell suspension for each group at $1 \times \mathrm{E}+06$ cells $/ \mathrm{mL}$, and put $10 \mathrm{ml}$ cell suspension in T75 flask (two flasks for each transduced construct). Treated one flask of each transduced construct group with DMSO and the other flask with $100 \mathrm{nM}$ TMP in PRMI1640 medium with $10 \%$ FBS for Jurkat cells and X-vivo 15 medium, 5\% heatinactivated AG serum-Sigma, $1 \times$ Pen/Strap, and $100 \mathrm{U} / \mathrm{mL}$ IL-2 for human pan-T. The killing efficacy was quantified by flow cytometry combined with BD liquid counting beads (BD Pharmingen Cat\# 335925) on day 1, day 2, and day 3 post treatment (three replicates per sample). On the day of flow analysis, first took $1 \mathrm{ml}$ cell culture into $1.5 \mathrm{~mL}$ Eppendorf tube from each flask, added $50 \mu \mathrm{L}$ BD liquid counting beads to each tube and span. Then removed medium and added $100 \mu \mathrm{L}$ BD staining buffer (BD Pharmingen Cat\# 554657) containing Pierce Recombinant Protein L, Biotinylated (Thermo Scientific Cat\# 29997) at 1:100 ratio. Incubated for $30 \mathrm{~min}$ at $4{ }^{\circ} \mathrm{C}$, then washed cells twice with $1 \mathrm{~mL}$ PBS. Removed PBS and added $100 \mu \mathrm{L}$ of BD staining buffer with PE Streptavidin (BD Pharmingen Cat\# 554061) as secondary (1:10 dilution) and incubate for $30 \mathrm{~min}$ at $4{ }^{\circ} \mathrm{C}$, then washed twice with PBS. Then removed buffer and resuspended cells and beads in $\mathrm{BD}$ staining buffer with Annexin V-Cy5 (Abcam Cat\# ab14150) incubated for 10min in dark at room temperature. Span and removed buffer. Then fixed cells with $1 \%$ paraformaldehyde in BD staining buffer for $60 \mathrm{~min}$ at room temperature. Then washed cells twice with $500 \mu \mathrm{L}$ BD staining buffer then resuspend in $200 \mu \mathrm{L}$ BD staining buffer for flow cytometry analysis. The elimination rate of GFP positive cells shown in the content of Figure 1 was calculated based on the following formula:

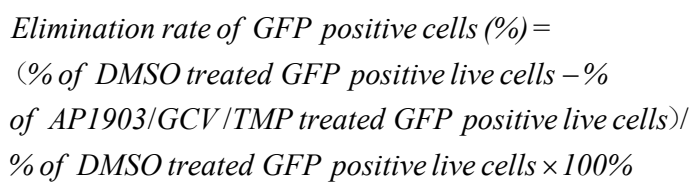

Elimination rate of GFP positive cells $(\%)=$ (\% of DMSO treated GFP positive live cells -\% of AP1903/GCV/TMP treated GFP positive live cells)/ $\%$ of DMSO treated GFP positive live cells $\times 100 \%$

During flow cytometry analysis, 50,000-100,000 cells 
were assayed for each replicate, and three replicates were analyzed for each sample. All flow cytometry based assays were repeated for more than three times in both Jurkat and human pan-T cells.

\section{Real-time PCR (TaqMan)}

RNA samples were extracted from different groups of cells (unactivated pan-T, mock pan-T, CD3/CD28 activated pan-T, $b E F 1 \alpha-C A R-e G F P$ transduced pan-T, $b E F 1 \alpha-C A R-$ $t B I D$ transduced pan-T, mock Jurkat, and $b E F 1 \alpha-C A R$ $t B I D$ transduced Jurkat) with QIAGEN RNeasy Mini Kit (QIAGEN, Cat\# 74104) by following the manufactory user manual. The RNA samples were then used to set up onestep real-time RT-PCR reactions by using SuperScript III platinum One-Step qRT-PCR kit (Invitrogen, Cat\# $11732020)$ at 384-well format by following the manufactory user manual. Predesigned TaqMan gene expression assays for a housekeeping gene, TBP, and few target genes, BID, $B C L 2 L 1$ and BCL2, were ordered from ThermoFisher Scientific. The real-time PCR (TaqMan) analysis were performed by using ViiA 7 real-time PCR system (Applied Biosystems, Cat\# 4453536).

\section{Statistical analysis}

The obtained Ct (Cycle Threshold) values were then used to calculate gene expression fold changes based on the standard real-time PCR data analysis process. Basically, took the average of the Ct values from the housekeeping gene $(T B P)$ and the gene being tested (BID, BCL2L1 and BCL2) in the experimental conditions (mock pan-T, CD3/CD28 activated pan-T, $b E F 1 \alpha-C A R-e G F P$ transduced pan-T, $b E F 1 \alpha-C A R-t B I D$ transduced pan-T, mock Jurkat, and bEF1 $\alpha-C A R-t B I D$ transduced Jurkat) and control condition (unactivated pan-T), returning 4 values: gene being tested experimental (TE), gene being tested control (TC), housekeeping gene experimental (HE), and housekeeping gene control (HC). Then calculated the differences between TE and HE (TE-HE) to obtain $\triangle$ CTE. And calculated the difference between TC and HC (TC-HC) to obtain $\triangle$ CTC. The Double Delta Ct value, $\Delta \Delta \mathrm{Ct}$, was then obtained by calculating the difference between $\triangle \mathrm{CTE}$ and $\triangle \mathrm{CTC}$ $(\Delta \mathrm{CTE}-\Delta \mathrm{CTC})$. Finally, the gene expression fold change was calculated by using formula $2^{\Delta \Delta C t}$. Real-time PCR experiment was repeated three times.

\section{Results}

\section{The tBID-based safety switch demonstrated slower but compatible killing efficacy with iCasp9 in Furkat cells}

In order to develop a novel, regulatable safety switch, we incorporated the pro-apoptotic role of tBID in cell death with the destabilizing feature of the mutant ecDHFR destabilizing domain to form the tBID-ecDHFR fusion protein (9). Theoretically, in the absence of TMP, the tBID-ecDHFR fusion protein would be unstable and quickly degraded. However, upon TMP treatment, the fusion protein should become stabilized and allow tBID to induce cell death. In order to test this hypothesis, tBID was fused to the $\mathrm{N}$-terminal ecDHFR destabilizing domain (NDHFR) mutant (R12Y/Y100I) (9) (Figure 1A) because the C-terminal ecDHFR destabilizing domain (CDHFR) mutant (N18T/A19V) had been demonstrated to be leaky in the absence of TMP treatment in previous literatures and our own experiment (Figure S1) (9). Since even low levels of tBID fusion protein could lead to undesired basal toxicity, the NDHFR-tBID fusion construct was chosen at this stage for efficacy assessment and subcloned into a lentiviral expression vector containing eGFP and puromycin resistance genes driven by the $M S C V$ promoter (Figure 1A). In the meantime, two well-established safety switches, iCasp9 and HSV-TK, and a NDHFR-fused humanized Renilla luciferase ( $b R L u c$ ) were also incorporated in the same lentiviral vectors as the positive and the negative controls, respectively (Figure 1A). Although the dosages of AP1903 and GCV to activate iCasp9 and HSVTK, respectively, were well established (3-6), the functional dosage of TMP was less-defined and further tested in the Jurkat cells transduced with lentivirus vectors $M S C V-e G F P$ $b R L u c$ and $M S C V-e G F P-t B I D(M O I=10)$. MOI $=10$ was chosen since it provided better transduction efficacy when compared with MOI $=1$ (Figure S1A). After two weeks of puromycin selection, the transduced Jurkat cells were treated with TMP at various concentrations $(0,100 \mathrm{nM}$, $1 \mu \mathrm{M}, 2 \mu \mathrm{M}$, and $5 \mu \mathrm{M})$ for two days. As shown in Figure $1 B$ and Figure S2, $48 \mathrm{~h}$ of TMP treatment at $100 \mathrm{nM}$ was able to increase cell death from $22.3 \%$ (no TMP treatment) to $73.6 \%$ in the $M S C V-e G F P-t B I D$ transduced cells without inducing additional cell death in the control-vector, $M S C V$ $e G F P-b R L u c$, transduced cells. However, TMP treatment at higher concentration $(2$ and $5 \mu \mathrm{M})$ induced additional cell death in the control group (Figure 1B, Figure S2B), 


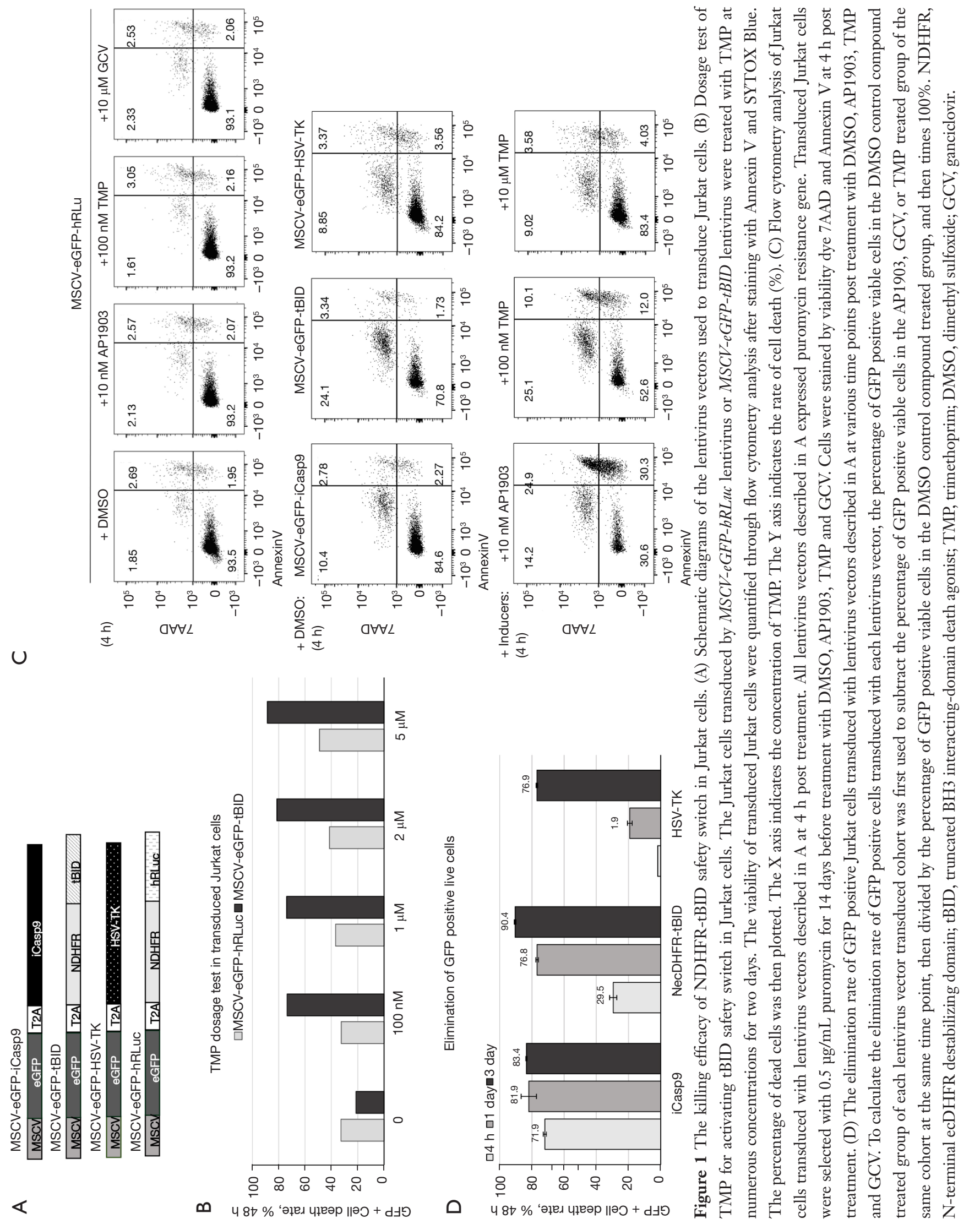


indicating potential toxicity at higher concentration. Therefore, the lowest concentration of TMP $(100 \mathrm{nM})$ was used in this study to activate the tBID-based safety switch.

To compare the efficacy of these constructs to induce cell death, the packaged lentiviral vectors were transduced into Jurkat cells at MOI $=10$. After two weeks of puromycin selection, the transduced Jurkat cells were treated with either dimethyl sulfoxide (DMSO) as the vehicle control reagent or $10 \mathrm{nM}$ AP1903 to activate iCasp9, 100nM TMP to activate tBID, and $10 \mu \mathrm{M}$ GCV to activate HSV-TK, respectively. To investigate cell viability affected by the treatment, the viability of the positively-transduced cells (GFP positive) was monitored and quantified through flow cytometry analysis after 7-amino-actinomycin D (7AAD, a DNA dye staining late apoptotic cells) and Annexin V (a marker binds to cell surfaces expressing phosphatidylserine and recognizes early apoptotic cells) staining at a serial time-points (4 h, day 1 and day 3). As shown in Figure $1 C$, at $4 \mathrm{~h}$ after different compound treatments, no viability change was detected in the control MSCV-eGFP-bRLuc-transduced Jurket cells treated with different small molecules or the DMSO-treated cells transduced by the various lentivirus preps. However, at the same time-point, strong cell death was induced in iCasp9 construct (MSCV-eGFP-iCasp9) transduced group, whereas only mild induction in cell death was observed in the cells transduced with $M S C V$ $e G F P-t B I D$ virus and very rare induction in cell death was observed in the MSCV-eGFP-HSV-TK transduced cells (Figure 1C,1D).

To further analyze and quantify the flow cytometry results on days 1 and 3, the elimination rate of the GFPpositive cells was calculated (Figure S3). As plotted in Figure 1D, acting as a fast-acting safety switch, iCasp9 was able to eliminate $71.9 \%$ GFP positive cells at $4 \mathrm{~h}$ after being activated by AP1903. On day 1 and day 3 post AP1903 treatment, the activated iCasp9 eliminated 81.1\% and $83.4 \%$ GFP-positive cells, respectively. On the other hand, the slow acting safety switch, HSV-TK, showed no obvious killing activity at $4 \mathrm{~h}$ after GCV treatment. However, it's killing effects started gradually, with 19.2\% elimination rate on day 1 and $76.9 \%$ elimination rate on day 3 post GCV treatment. The NDHFR-tBID safety switch demonstrated a moderate killing effect that fell into the range between iCasp9 and HSV-TK. The elimination rates of the NDHFR-tBID engineered Jurkat cells with TMP treatment at $4 \mathrm{~h}$, day 1 and day 3 time points were $29.5 \%$, $76.8 \%$ and $90.4 \%$, respectively (Figure $1 D$ ).

\section{NDHFR-tBID activity was attenuated in buman pan-T cells}

After validating the efficacy of NDHFR-tBID safety switch in the Jurkat cells, a T-cell leukemia cell line, we incorporated the NDHFR-tBID and a control eGFP expression cassette, respectively, into a CAR expression vector under the control of $h E F 1 \alpha$ promoter to compare and assess their killing activities in primary human pan-T cells (Figure 2A). After these constructs were further packaged into lentiviral vectors, to compare the efficacy of safety switch side by side in both human pan-T and Jurkat cells, the lentiviral vectors expressing the engineered CAR constructs were transduced into both cell types at MOI $=10$. The expression of transduced CAR transgenes was confirmed by using flow cytometry via Protein L staining which binds to immunoglobulin kappa light chains. Lentiviral vector $b E F 1 \alpha-C A R-t B I D$ had $13 \%$ transduction rate in human pan-T cells and $52 \%$ transduction rate in Jurkat cells (Figure S4). Lentiviral vector bEF1 $\alpha-C A R$ $e$ GFP had $17 \%$ transduction rate in human pan-T cells (Figure S4). Lower transduction rate was observed in human pan-T cells for the same lentiviral vector.

On day 7 post transduction, lentivirus transduced human pan-T cells, Jurkat cells, along with mock control cells (cells went through transduction procedure without adding lentivirus) for both cell types were treated with either DMSO or $100 \mathrm{nM}$ TMP to induce the apoptotic activity of NDHFR-tBID. The efficacy of eliminating CARpositive cells was measured through Protein L staining and flow cytometry at $72 \mathrm{~h}$ post induction. As shown in Figure $2 B$, at $72 \mathrm{~h}$ after induction, the CAR-positive Jurkat cells that were transduced with $b E F 1 \alpha-C A R-t B I D$ were reduced from $47.4 \%$ in DMSO treated group to $17.9 \%$ in TMP treated group, while no cytotoxicity was detected in the mock control group. This indicated strong killing efficacy of NDHFR-tBID in eliminating CAR positive Jurkat cells. However, the CAR-positive human pan-T cells transduced with $b E F 1 \alpha-C A R-t B I D$ were only reduced from $13.3 \%$ in DMSO treated group to $~ 10 \%$ in TMP treated group during the same duration of treatment (Figure $2 B$ ). Therefore, NDHFR-tBID demonstrated much weaker killing potency in human pan-T cells than in Jurkat cells. To investigate the reasons why NDHFR-tBID lost the killing potency in human pan-T, real-time PCR (TaqMan) was first performed to validate the BID expression levels in human pan-T and Jurkat cells. As indicated in Figure $2 C$, the BID expression level in human pan-T cells transduced with 

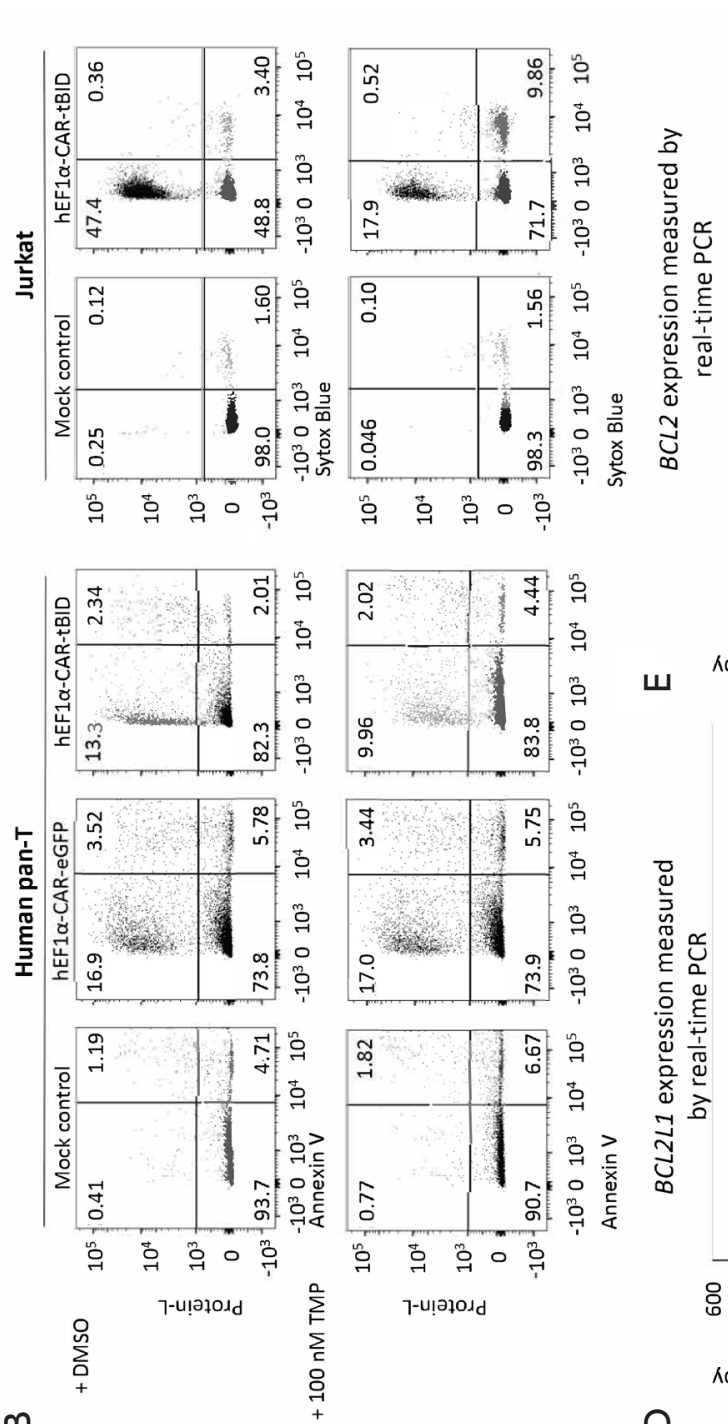

$\infty$

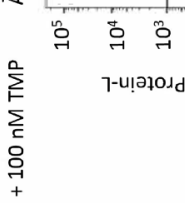

山
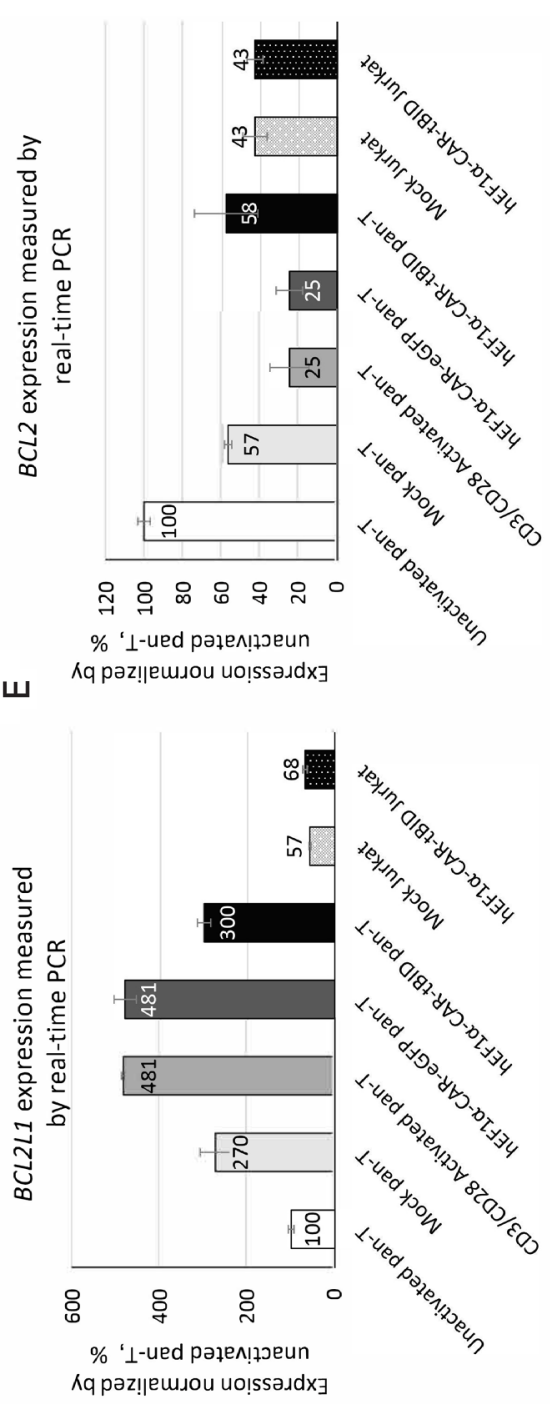

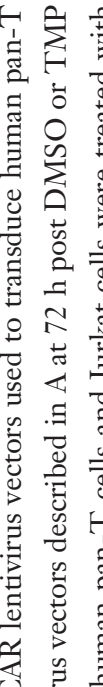

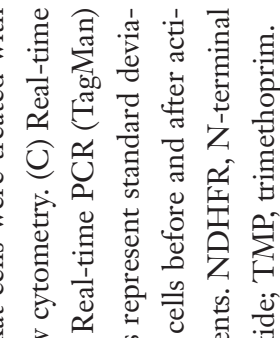

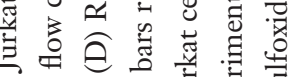

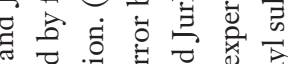

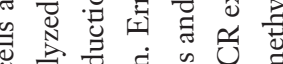
ज़ 志

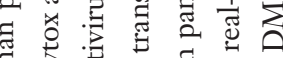
का 0 政

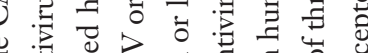
氙 प्र

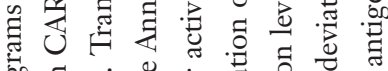

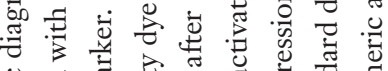

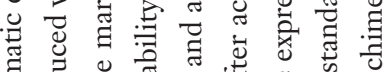

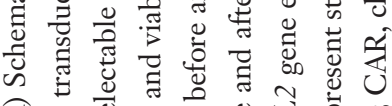

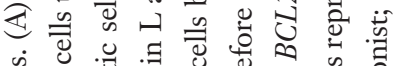

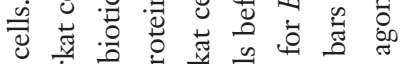

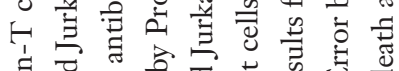

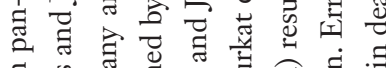

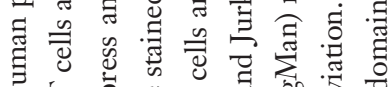
H .

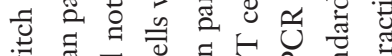

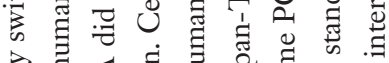

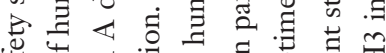

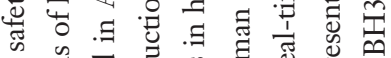

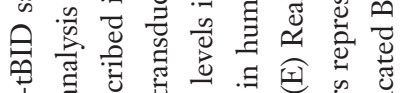

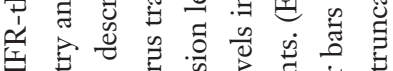

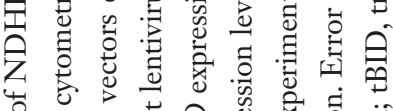
प U ग

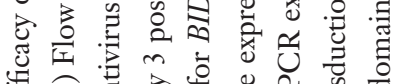

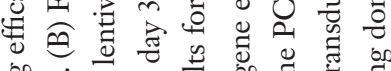

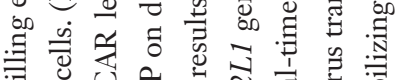

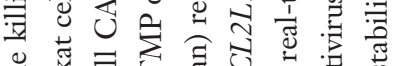

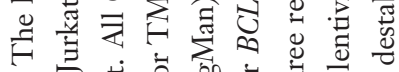

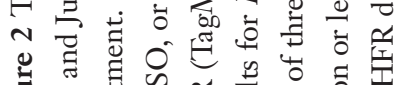

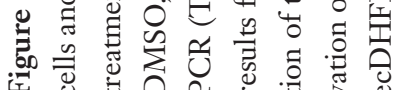


bEF1 $\alpha$-CAR-tBID lentivirus was 6.6-fold (655.6/100) higher than unactivated pan-T, 2.4-fold (655.6/277.8) higher than mock transduced pan-T cells, and 10.3-fold (655.6/63.9) higher than $b E F 1 \alpha-C A R-e G F P$ lentivirus transduced pan-T cells. Due to the fact that the real-time PCR assay was not able to distinguish endogenous $B I D$ and transduced $t B I D$ expression, significant endogenous $B I D$ expression was detected in mock transduced Jurkat cells. However, transduction of $b E F 1 \alpha-C A R-t B I D$ lentivirus provided additional level of BID expression in Jurkat cells. The enhanced $B I D$ expression in $b E F 1 \alpha-C A R$ - $t B I D$ lentivirus transduced pan-T cells compared to mock transduced pan-T cells $(655.6 \%-277.8 \%=377.8 \%)$ and the enhanced $B I D$ expression in $b E F 1 \alpha-C A R-t B I D$ lentivirus transduced Jurkat cells compared to mock transduced Jurkat cells $(2,500 \%-2,030.6 \%=469.4 \%)$ were close. Therefore, the lack of killing potency of NDHFR-tBID safety switch in human pan- $T$ cells was not due to vector expression failure.

\section{$B C L-X L$ and BCL-2 in buman pan-T cells may inbibit the activity of NDHFR-tBID safety switch}

In order to further explore the causes for NDHFR-tBID attenuation in human pan- $\mathrm{T}$ cells, we looked for other potential inhibitors of tBID in human pan-T. BCL-XL and BCL-2 are BCL-2 family members that prevent apoptosis induced by a variety of death stimuli $(8,12,13)$. It has been shown that anti-CD3 and anti-CD28 coactivation of $\mathrm{T}$ cells could strongly upregulate BCL-XL expression to promote survival of activated $\mathrm{T}$ cells $(14,15)$. In addition multiple lines of established studies have shown that BCL-XL and BCL-2 inhibits the apoptotic changes induced by tBID $(8,13)$, we then hypothesize that BCL-2 and upregulated BCL-XL in activated human pan-T cells could contribute to the attenuation of NDHFR-tBID safety switch. To test this hypothesis, real-time PCR (TaqMan) was performed to validate the gene expression levels of $B C L 2$ (encodes BCL-2) and BCL2L1 (encodes BCL-XL) in human pan-T cells and Jurkat cells pre- and post-transduction. As shown in Figure 2D, upon activation by anti-CD3/anti-CD28 Dynabeads or viral transduction, the BCL2L1 expression was elevated by 3-5 folds in human pan-T cells. However, the BCL2L1 expression was low and stable in Jurkat cells after transduction. After activation or transduction, human pan-T cells had 4-7 folds higher BCL2L1 than Jurkat cells (Figure 2D). The BCL2 expression was slightly higher ( $\sim 1.4$ folds) in human pan-T cells than in Jurkat cells after the transduction of $b E F 1 \alpha-C A R-t B I D$ lentivirus
(Figure 2E). High level of BCL2L1 was detected when NDHFR-tBID activity was attenuated in activated human pan-T cells. Overall, our observation suggests that differential expression of pro- or anti-apoptotic pathway components between different cell types or preparations may affect the performance of our safety switch in the tested cells.

\section{Improving the potency of tBID safety switch in human pan-T cells through mutating in BH3 domain and fusing with CDHFR}

Because elevated levels of BCL-XL and BCL-2 expression in human pan- $\mathrm{T}$ might result in the low efficacy of NDHFR-tBID safety switch, to address these issues, we inspected and fine-tuned the safety switch system by increasing tBID expression and disrupting the inhibitory influence of BCL-XL and BCL-2 in human pan-T cells.

The reported leakage effect of C-terminal ecDHFR destabilizing domain (CDHFR) mutant (N18T/ A19V) (9) (Figure S1) has hampered the initial attempt to fuse it with tBID. However the CDHFR mutant offered higher levels of fusion protein expression upon TMP induction when compared with NDHFR fusion protein (9) (Figure S1). We then fused CDHFR to the C-terminus of tBID (tBID-CDHFR) with the expectation of using stronger tBID expression to overcome the inhibitory effects of BCL-XL and BCL-2 (Figure 3A). The published studies in recombinant BID proteins had identified a mutant (M97A/D98A) that specifically disrupted the interaction between BID and BCL-2 $(2,7)$. We therefore incorporated the M97A/D98A mutation to the NDHFR-tBID construct, $b E F 1 \alpha-C A R-t B I D(M)$, aiming to attenuate the inhibitory influence of BCL-2 on tBID safety switch (Figure $3 A$ ). Lentiviral constructs carrying these modifications were packaged (Figure $3 A$ ) and transduced into human pan-T cells at $\mathrm{MOI}=10$. Due to the low transduction efficiency observed in human pan- $\mathrm{T}$ cells, the positively transduced cells expressing the CAR transgene were enriched by Protein L beads prior to further treatment (Figure S5). To validate the killing efficacy of the modified tBID safety switches, the transduced human pan-T cells after Protein $\mathrm{L}$ beads enrichment were treated with either DMSO or $100 \mathrm{nM}$ TMP. The efficacy of eliminating CAR-positive cells was measured by flow cytometry via Protein L staining at $72 \mathrm{~h}$ post compound treatment (Figure $3 B$ ). As demonstrated in Figure $3 B$, addition of the M97A/ D98A mutation to tBID [ $b E F 1 \alpha-C A R-t B I D(M)]$ slightly 
A

hEF1 $\alpha$-CAR-tBID

\begin{tabular}{|c|c|c|c|c|}
\hline hEF $1 \alpha$ & CAR & T2A & NDHFR & $t \mathrm{BID}$ \\
\hline \multicolumn{5}{|c|}{ F1 $\alpha$-CAR-tBID (M) } \\
\hline hEF1 $\alpha$ & CAR & T2A & NDHFR & tBID (M) \\
\hline
\end{tabular}

hEF1 $\alpha$-CAR-tBID-CDHFR

\begin{tabular}{|l|l|l|l|l|}
\hline hEF1 $\alpha$ & CAR & T2A & TBID & CDHFR \\
\hline
\end{tabular}

hEF1 $\alpha$-CAR-eGFP

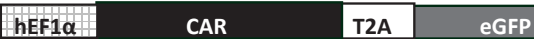

B

(72 h)

+ DMSO
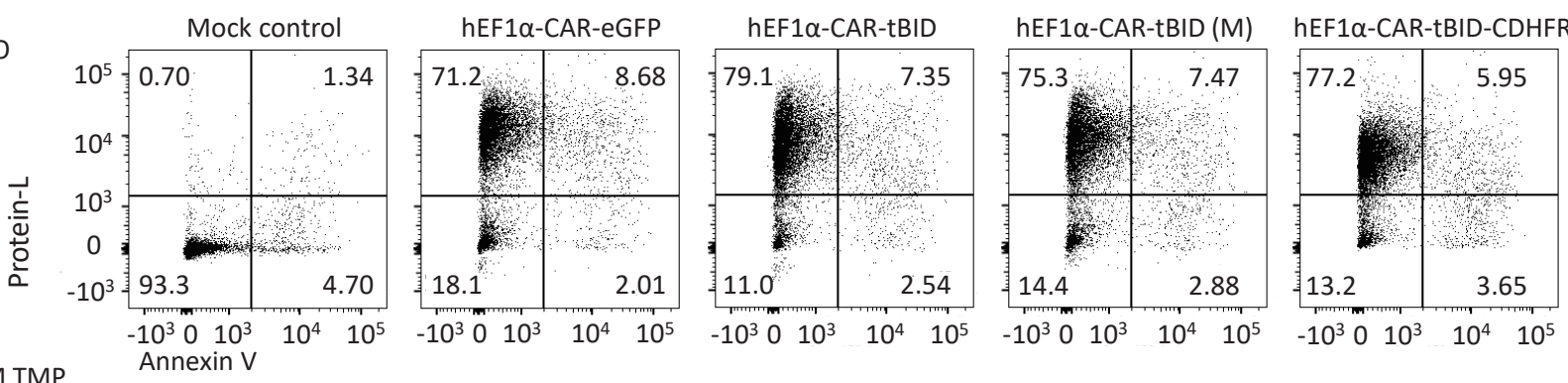

+100 nM TMP
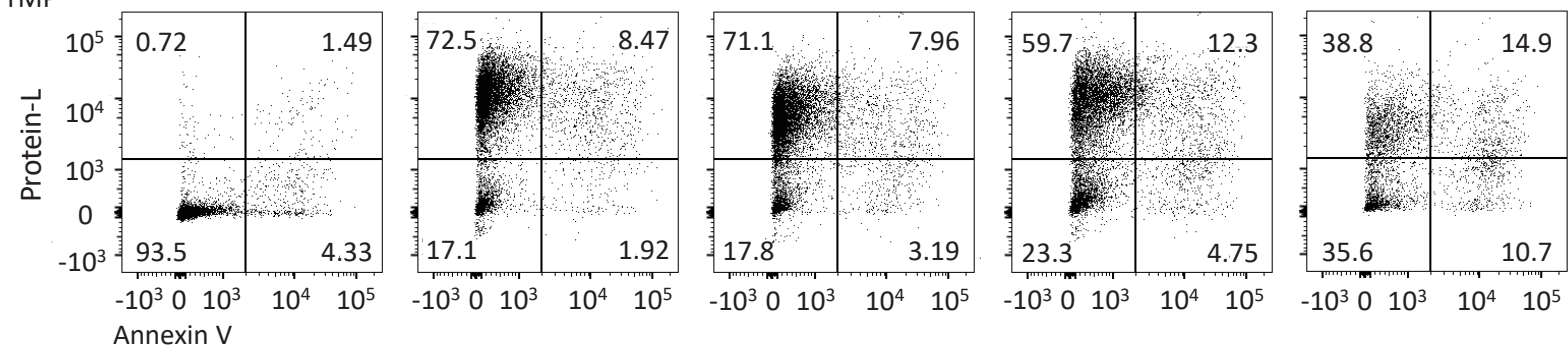

C

Cell count normalized by DMSO $(\%)=\begin{aligned} & \text { Protein-L positive live cells/Beads } \\ & (\text { TMP })\end{aligned} / \begin{aligned} & \text { Protein-L positive live cells/Beads } \\ & \text { (DMSO) }\end{aligned}$ X 100\%

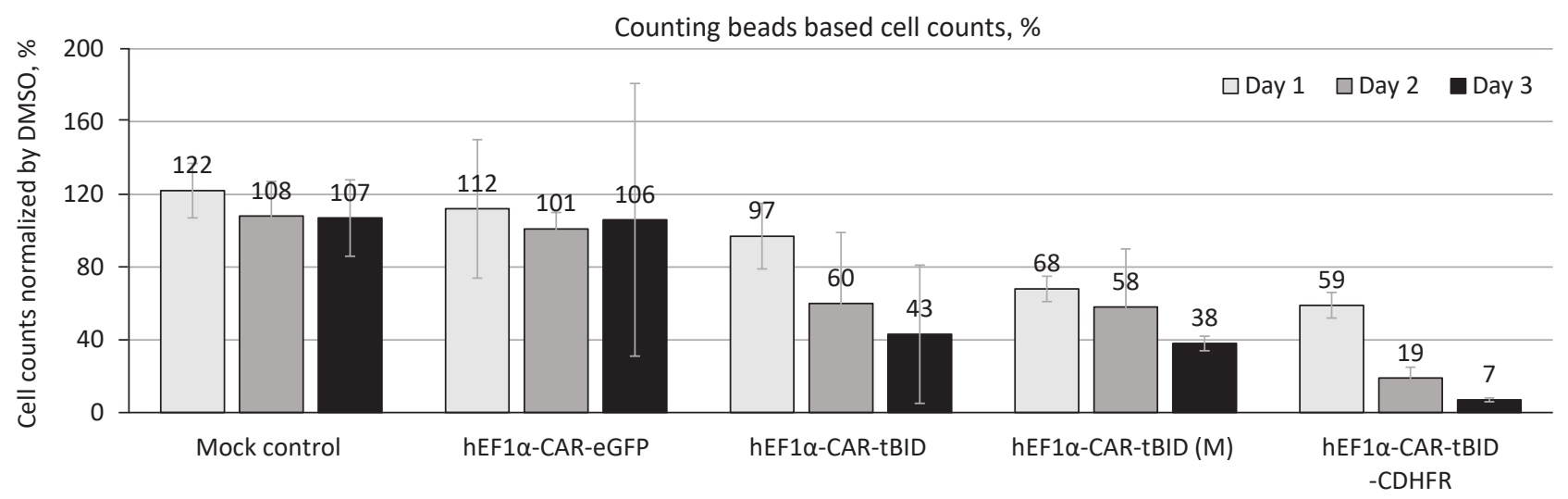

Figure 3 The killing efficacy of modified tBID safety switches in human pan-T cells. (A) Schematic diagrams of the CAR lentivirus constructs with modified tBID safety switches used to transduce human pan-T cells. (B) Flow cytometry analysis of human pan-T cells transduced with CAR lentivirus vectors described in A at $72 \mathrm{~h}$ post DMSO or TMP treatment. All CAR lentivirus vectors described in A did not express any antibiotic selectable marker. Transduced human pan-T cells were treated with DMSO or TMP on day 3 post lentivirus transduction. Cells were stained by Protein L and viability dye Annexin V and analyzed by flow cytometry. (C) Viable cell count quantified by cell counting beads and flow cytometry analysis. Human pan-T cells transduced with lentivirus described in A were treated with DMSO or TMP on day 3 post transduction. The cells were stained with Protein L and Annexin $\mathrm{V}$ at various time points post treatment and quantified by cell counting beads during flow cytometry analysis. The cell counts in TMP treated groups were normalized by DMSO treat group and converted into percentage. tBID, truncated $\mathrm{BH} 3$ interacting-domain death agonist; CAR, chimeric antigen receptor; DMSO, dimethyl sulfoxide; TMP, trimethoprim. 
enhanced the killing potency of NDHFR-tBID by reducing CAR positive population from $75.3 \%$ (DMSO-treated) to $59.7 \%$ (TMP-treated). On the other hand, the nonmodified NDHFR-tBID ( $b E F 1 \alpha-C A R-t B I D)$ only reduced CAR positive population from $79.1 \%$ (DMSO treated) to $71.1 \%$ (TMP treated). Fusing CDHFR with tBID provided improved potency as TMP treated $b E F 1 \alpha-C A R-t B I D-$ CDHFR transduced cells reduced CAR-positive population to $38.8 \%$ compared with $77.2 \%$ in DMSO treated group. While analyzing the flow cytometry data, it was noted that the total viable cell number was significantly reduced in bEF1 $\alpha-C A R-t B I D-C D H F R$ transduced cells after TMP treatment. We then combined cell counting beads along with flow cytometry analysis to quantify the killing efficacy of modified tBID safety switches more precisely. As shown in Figure 3C, DMSO- or $100 \mathrm{nM}$ TMP-treated transduced cells were stained with Protein $\mathrm{L}$ and viability dye at various time points after treatment, then counted with cell counting beads through flow cytometry for the CAR positive live cell number. While the control vector $b E F 1 \alpha-C A R-e G F P$ transduced human pan- $T$ cells had the same viable CAR positive cells as mock control, the non-modified NDHFRtBID construct, $b E F 1 \alpha-C A R-t B I D$, transduced viable CAR positive cells were gradually removed with $97 \%$ viable CAR-positive cells on day 1, 60\% viable CAR-positive cells on day 2 , and only $43 \%$ viable CAR-positive cells detected on day 3 post TMP treatment. Incorporating M97A/D98A to tBID [bEF1 $\alpha-C A R-t B I D(M)]$ slightly improved killing efficacy with detection of $68 \%$ viable CAR-positive cells on day-1, $58 \%$ viable CAR-positive cells on day-2, and $38 \%$ viable CAR-positive cells on day- 3 post TMP treatment. The most robust killing potency was observed in $b E F 1 \alpha-$ CAR-tBID-CDHFR transduced cells that $59 \%$ viable CAR positive cells on day-1, $19 \%$ viable CAR positive cells on day-2, and only $7 \%$ viable CAR positive cells on day- 3 could be detected post TMP treatment. These data indicated that fusing CDHFR with tBID significantly enhanced the killing efficacy while adding M97A/D98A mutations to tBID only slightly improved the killing efficacy.

\section{Discussion}

In this study, we developed a safety switch based on the activity of tBID. In order to conveniently regulate the apoptotic activity of tBID, we took advantage of the cellular degradation machinery by fusing tBID to an engineered ecDHFR-derived destabilizing domain (9). The fusion of ecDHFR destabilizing domain to tBID confers the instability to entire fusion protein and causes rapid degradation. Application of ecDHFR specific inhibitor, TMP, stabilizes the destabilizing domain and the fusion tBID protein resulted in the activation of tBID apoptotic activity. The $\mathrm{N}$-terminal destabilizing domain (mutant R12Y/Y100I) was first chosen to fuse with tBID in our study due to its high efficacy demonstrated by Iwamoto et al. (9). The high potency of this NDHFR-tBID safety switch was validated and proven in Jurkat cells. However, the NDHFRtBID safety switch failed to induce cell death in human pan-T cells. The real-time PCR experiment measuring $B I D$ expression has indicated the successful expression of NDHFR-tBID safety switch from the transduced lentivirus (Figure 2C). Therefore, other mechanisms that could inhibit NDHFR-tBID safety switch function were considered. As a death ligand, BID activates the mitochondrial membranebound receptor BAX through heterodimerizing the domain of BID with the BH1 domain of BAX (7). As a pro-apoptotic BCL-2 family member, BAX promotes cell death after being activated by BID through forming large oligomers that permeabilize the outer mitochondrial membrane, thereby committing cells to apoptosis. BCL-XL and BCL2 are anti-apoptotic BCL-2 family members that prevent apoptosis induced by a variety of death stimuli $(8,12,13)$. The BH1 domain of BCL-2 also interacts with the BH3 domain of BID (7), with heterodimerization between BID and BCL-2 inhibiting apoptosis (7). Previous studies have also shown that human T cells overexpressing BCL-2 were resistant to cell death (16). BAX also heterodimerizes with BCL-2 or BCL-XL (17). BCL-XL is structurally similar to $\mathrm{BAX}$ and competes with $\mathrm{BAX}$ for activation through interaction with membranes, BID, or BID activated BAX, thereby inhibiting BAX binding to membranes, oligomerization, and membrane permeabilization (18). It has been shown that anti-CD 3 and anti-CD28 coactivation of $\mathrm{T}$ cells could strongly upregulate BCL-XL expression to promote survival of activated $T$ cells $(14,15)$. Since multiple lines of established studies have shown that BCL$\mathrm{XL}$ and BCL-2 inhibits the apoptotic changes induced by tBID $(8,13)$, we then asked if the expression levels of BCL-2 and BCL-XL were upregulated in activated or lentivirus transduced human pan-T cells. The real-time PCR we performed demonstrated that human pan-T cells had 4-7-fold higher BCL2L1 gene expression and slightly higher (1.4-fold) BCL2 gene expression than Jurkat cells after activation or transduction. Therefore, both BCL2 and BCL-XL could serve as inhibitors of tBID based safety switch in activated human pan-T cells. This limits 
the application of NDHFR-tBID in cells that have high levels of BCL-XL and BCL-2 expression. To overcome this limitation, we incorporated mutations (M97A/D98A) that have been demonstrated to disrupt the BCL-2 inhibition on tBID $(2,7)$. The resulted modified NDHFR-tBID (M) safety switch did display stronger killing potency compared to non-modified NDHFR-tBID (Figure 3B,3C). However more striking improvement was achieved by switching the $\mathrm{N}$-terminal destabilizing domain (mutant R12Y/Y100I) to the C-terminal destabilizing domain (mutant N18T/ A19V) (9). C-terminal destabilizing domain fusion proteins have demonstrated higher expression levels upon TMP treatment than $\mathrm{N}$-terminal destabilizing domain fusion proteins (Figure S1) (9), however the leakage effects from C-terminal destabilizing domain hindered the initial attempt to fuse it with tBID in Jurkat cells (Figure S1). The C-terminal destabilizing domain fused tBID safety switch tBID-CDHFR has demonstrated robust killing efficacy upon TMP treatment in human pan-T cells (Figure 3B,3C), indicating stronger tBID expression could mitigate some inhibitory effects of BCL-XL and BCL-2. Considering the potential basal toxicity of the C-terminal destabilizing domain, we did not incorporate the M97A/D98A mutations to tBID-CDHFR safety switch. Although the potency of tBID based safety switch was improved after fusing tBID with CDHFR or incorporating M97A/D98A mutations under our in vitro condition, the inhibitory effects of BCL$\mathrm{XL}$ and BCL-2 on tBID safety switch need to be considered when applying to other cell types.

The optimal safety switch will be fast-acting, with no or low immunogenicity, no or low basal toxicity and induced through a non-toxic small molecule. The tBID-CDHFR safety switch achieved a killing outcome between two clinically-validated safety switches, iCasp9 and HSV-TK, and enabled to kill $~ 90 \%$ transduced human pan-T cells in 3 days after $100 \mathrm{nM}$ TMP treatment in our in vitro assay (Figure 3C). The immunogenicity of ecDHFR-derived destabilizing domain will need to be determined through further in vivo studies and might potentially be mitigated through codon optimization.

Since we compared iCasp9 and tBID based safety switch in Jurkat cells, we planned to do the same comparation of these safety switches in human pan-T cells side by side as well. However, despite many attempts, we were not able to package $b E F 1 \alpha-C A R-i C a s p 9$ into enough lentiviral vector due to low viability of the HEK293 cells used to package this construct (Table S1). There could be some element inhibiting lentiviral packaging in this vector which requires further modification or expression of CAR-iCasp 9 during lentiviral vector packaging was detrimental to HEK293 cell's viability. We therefore removed this vector from testing list in human pan-T cells. No such toxicity was observed when packaging tBID constructs into lentiviral vector during this study. TMP as a very well-established antibiotic used to treat bacterial infections has been demonstrated to stabilize ecDHFR in a rapid, reversible and dose-dependent format.

\section{Conclusions}

In summary we have developed a fast-acting tBID based safety switch with low basal toxicity that could be regulated through a commercially available, inexpensive, and BBB permeable compound, TMP. Further investigation or characterization for applications of the tBID-based safety switch in vivo should be worthy to pursuit.

\section{Acknowledgments}

The authors would like to thank Lili Yue and Qingwen Cheng for sharing experimental reagents.

Funding: None.

\section{Footnote}

Reporting Checklist: The authors have completed the MDAR reporting checklist (available at http://dx.doi.org/10.21037/ atm-21-443).

Data Sharing Statement: Available at http://dx.doi. org/10.21037/atm-21-443

Conflicts of Interest: All authors have completed the ICMJE uniform disclosure form (available at http:// dx.doi.org/10.21037/atm-21-443). JL, CML, and SW are employees at Amgen Inc. PC and KJL were employed by Amgen Inc. while working on the study. All authors owned Amgen shares when the experiments were carried out. However, these do not alter the authors' adherence to all journal policies on sharing data and materials.

Ethical Statement: The authors are accountable for all aspects of the work in ensuring that questions related to the accuracy or integrity of any part of the work are appropriately investigated and resolved. Human T-cell samples were purchased anonymously from the vendor and considered exempt from IRB approval. 
Open Access Statement: This is an Open Access article distributed in accordance with the Creative Commons Attribution-NonCommercial-NoDerivs 4.0 International License (CC BY-NC-ND 4.0), which permits the noncommercial replication and distribution of the article with the strict proviso that no changes or edits are made and the original work is properly cited (including links to both the formal publication through the relevant DOI and the license). See: https://creativecommons.org/licenses/by-nc-nd/4.0/.

\section{References}

1. Bonini C, Ferrari G, Verzeletti S, et al. HSV-TK gene transfer into donor lymphocytes for control of allogeneic graft-versus-leukemia. Science 1997;276:1719-24.

2. Oh KJ, Barbuto S, Pitter K, et al. A membrane-targeted BID BCL-2 homology 3 peptide is sufficient for high potency activation of BAX in vitro. J Biol Chem 2006;281:36999-7008.

3. Beltinger C, Fulda S, Kammertoens T, et al. Herpes simplex virus thymidine kinase/ganciclovir-induced apoptosis involves ligand-independent death receptor aggregation and activation of caspases. Proc Natl Acad Sci U S A 1999;96:8699-704.

4. Straathof KC, Pulè MA, Yotnda P, et al. An inducible caspase 9 safety switch for T-cell therapy. Blood 2005;105:4247-54.

5. Di Stasi A, Tey SK, Dotti G, et al. Inducible apoptosis as a safety switch for adoptive cell therapy. N Engl J Med 2011;365:1673-83.

6. Gargett T, Brown MP. The inducible caspase-9 suicide gene system as a "safety switch" to limit on-target, offtumor toxicities of chimeric antigen receptor T cells. Front Pharmacol 2014;5:235.

7. Wang K, Yin XM, Chao DT, et al. BID: a novel BH3 domain-only death agonist. Genes Dev 1996;10:2859-69.

8. Li H, Zhu H, Xu CJ, et al. Cleavage of BID by caspase 8

Cite this article as: $\mathrm{Lu} \mathrm{J}$, Collins $\mathrm{P}$, Lee $\mathrm{KJ}, \mathrm{Li} \mathrm{CM}$, Wang S. In vitro assessment of an engineered tBID-based safety switch system in human T lymphocytes. Ann Transl Med 2021;9(22):1641. doi: 10.21037/atm-21-443 mediates the mitochondrial damage in the Fas pathway of apoptosis. Cell 1998;94:491-501.

9. Iwamoto M, Björklund T, Lundberg C, et al. A general chemical method to regulate protein stability in the mammalian central nervous system. Chem Biol 2010;17:981-8.

10. Sasso SP, Gilli RM, Sari JC, et al. Thermodynamic study of dihydrofolate reductase inhibitor selectivity. Biochim Biophys Acta 1994;1207:74-9.

11. Mauldin RV, Carroll MJ, Lee AL. Dynamic dysfunction in dihydrofolate reductase results from antifolate drug binding: modulation of dynamics within a structural state. Structure 2009;17:386-94.

12. Merry DE, Korsmeyer SJ. Bcl-2 gene family in the nervous system. Annu Rev Neurosci 1997;20:245-67.

13. Youle RJ, Strasser A. The BCL-2 protein family: opposing activities that mediate cell death. Nat Rev Mol Cell Biol 2008;9:47-59.

14. Boise LH, Minn AJ, Noel PJ, et al. CD28 costimulation can promote $T$ cell survival by enhancing the expression of Bcl-XL. Immunity 1995;3:87-98.

15. Mueller DL, Seiffert S, Fang W, et al. Differential regulation of bcl-2 and bcl-x by $\mathrm{CD} 3, \mathrm{CD} 28$, and the IL-2 receptor in cloned CD4+ helper T cells. A model for the long-term survival of memory cells. J Immunol 1996;156:1764-71.

16. Charo J, Finkelstein SE, Grewal N, et al. Bcl-2 overexpression enhances tumor-specific T-cell survival. Cancer Res 2005;65:2001-8.

17. Oltvai ZN, Milliman CL, Korsmeyer SJ. Bcl-2 heterodimerizes in vivo with a conserved homolog, Bax, that accelerates programmed cell death. Cell 1993;74:609-19.

18. Billen LP, Kokoski CL, Lovell JF, et al. Bcl-XL inhibits membrane permeabilization by competing with Bax. PLoS Biol 2008;6:e147. 


\section{Supplementary}

A

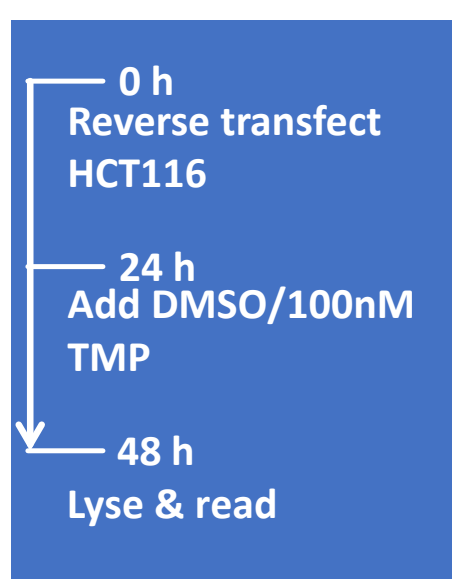

C

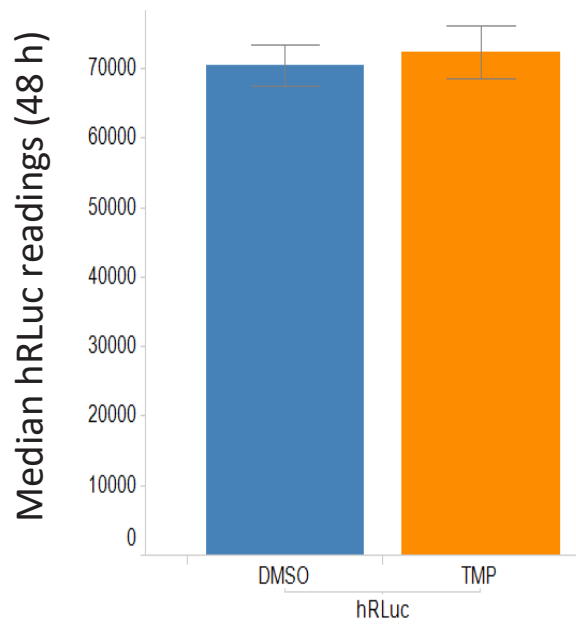

B

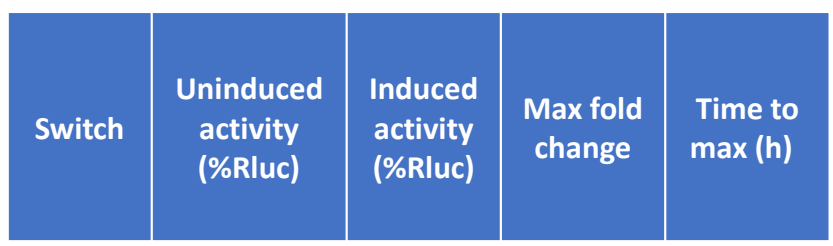

\begin{tabular}{|l|l|l|l|l|}
\hline NDHFR & $5 \%$ & $24 \%$ & 6 & 12 \\
\hline CDHFR & $34 \%$ & $91 \%$ & 3 & 12 \\
\hline
\end{tabular}
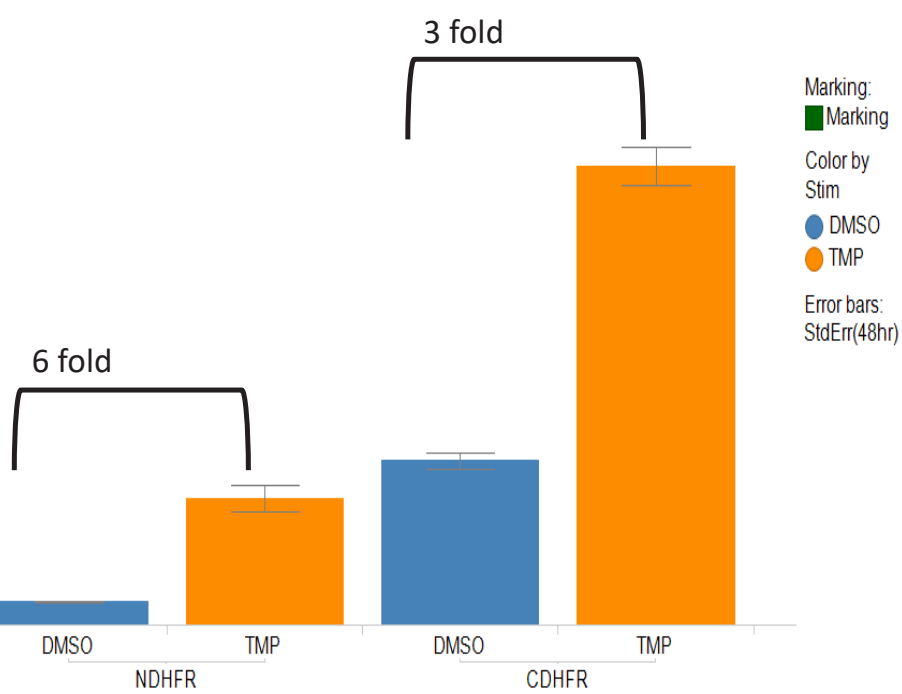

Figure S1 Compared the regulation efficacy and potency of NDHFR and CDHFR in HCT116 cells. (A) Outline of the experiment procedure. (B) Table to compare the efficacy and potency of NDHFR and CDHFR. (C) Bar chart of hRLuc readings at $48 \mathrm{~h}$ post TMP or DMSO (vehicle) treatment. 
A

GFP positive cell rate after MSCV-eGFP-hRLuc transduction

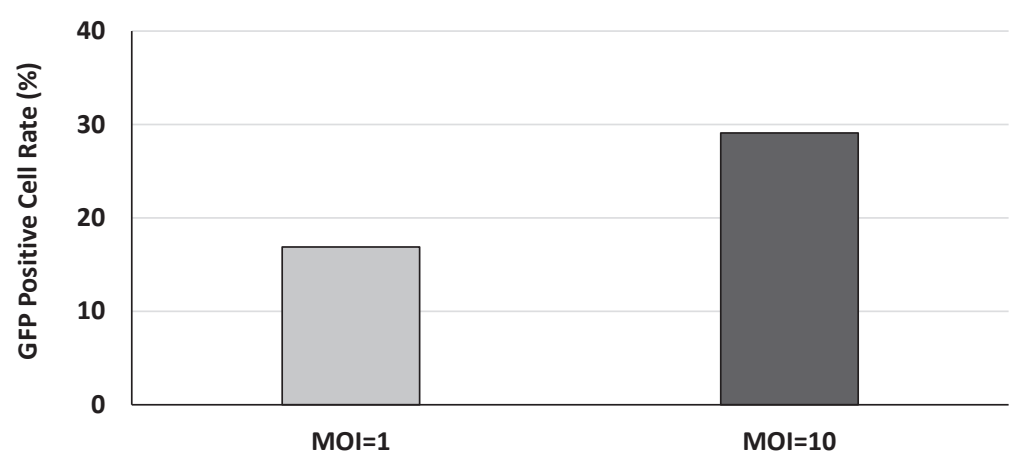

B

MSCV-eGFP-hRLuc (MOI 10)
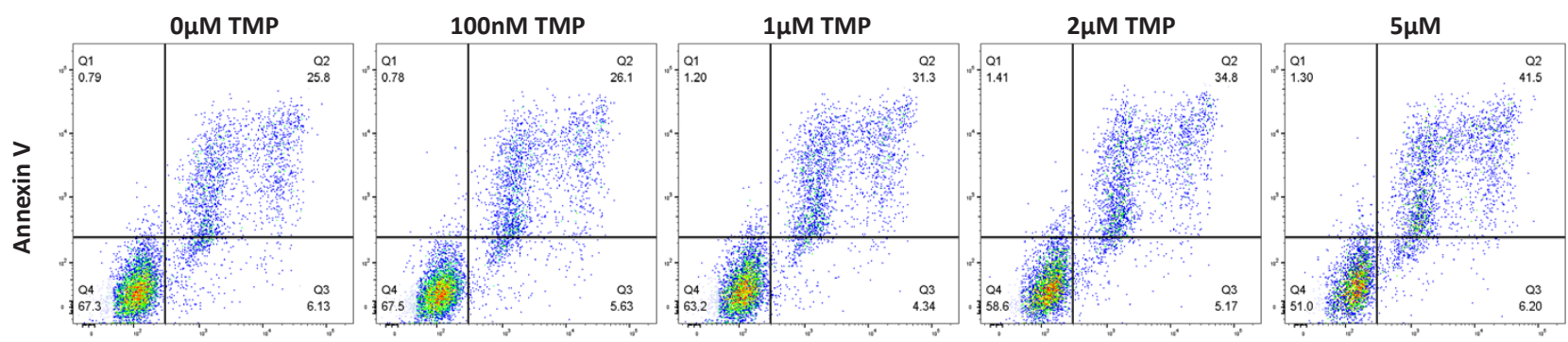

SYTOX Blue

MSCV-eGFP-tBID (MOI 10)
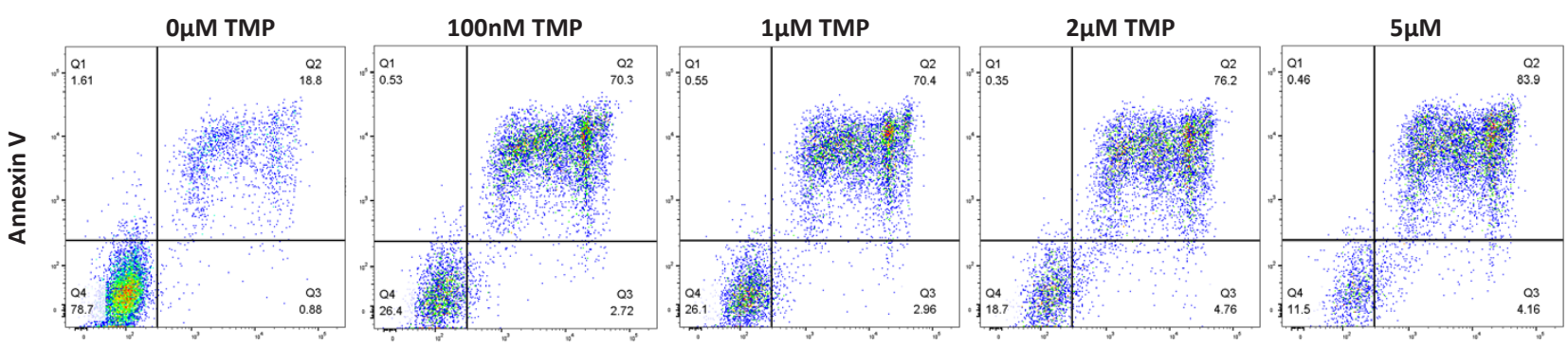

SYTOX Blue

Figure S2 Optimization of lentivirus transduction MOI and TMP dosage. (A) Comparison of lentivirus transduction efficacy in Jurkat cells at MOI 1 and MOI 10 by using $M S C V-e G F P-b R L u c$. The transduction efficacy was determined by the rate of GFP positive cells obtained from flow cytometry analysis. (B) Dosage test of TMP for activating tBID safety switch in Jurkat cells. The Jurkat cells transduced by $M S C V-e G F P-b R L u c$ lentivirus or $M S C V-e G F P-t B I D$ lentivirus were treated with TMP at numerous concentrations for two days. The viability of GFP positive transduced Jurkat cells were quantified through flow cytometry analysis after staining with Annexin $\mathrm{V}$ and SYTOX Blue. 

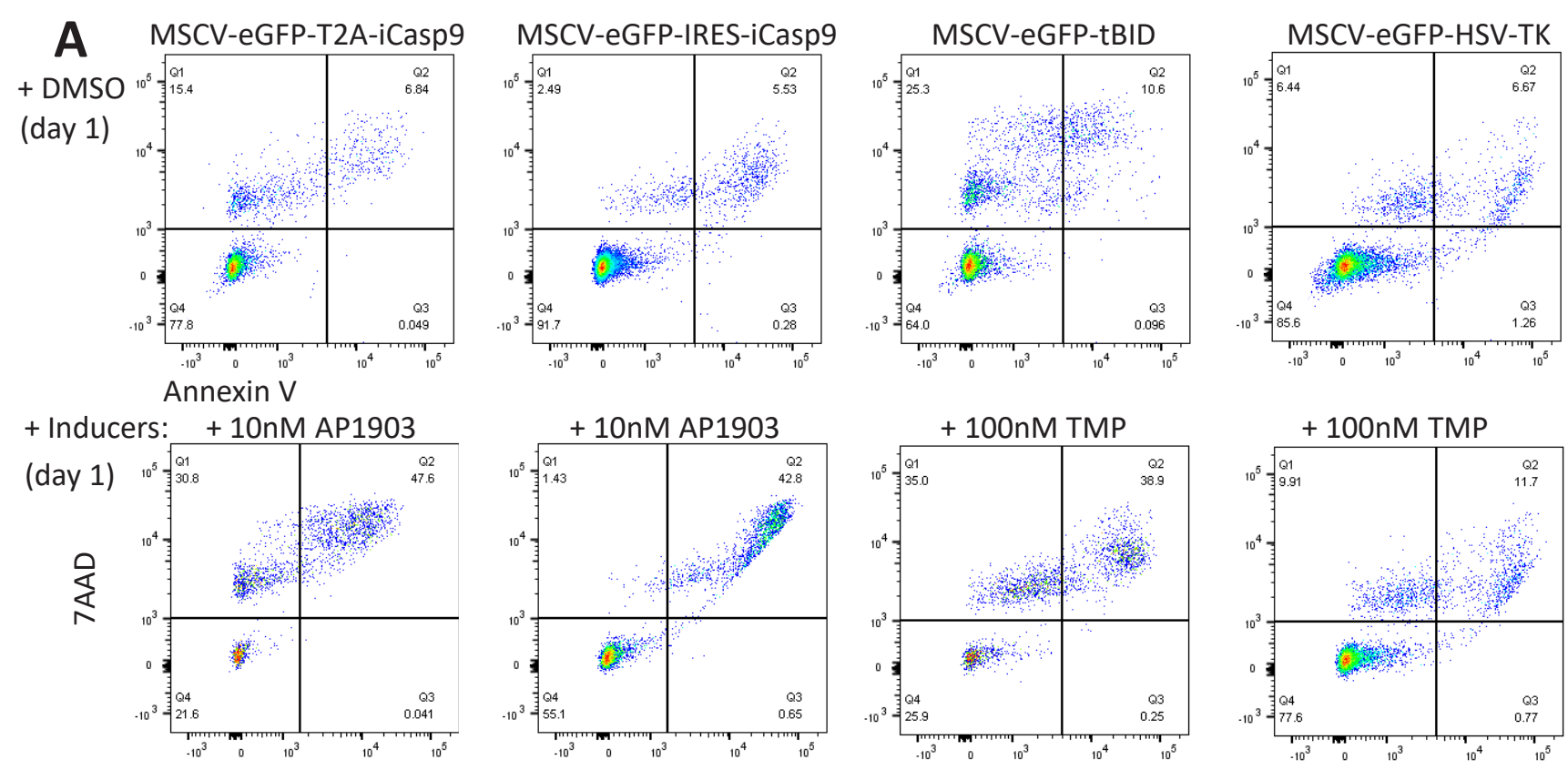

Annexin $\mathrm{V}$

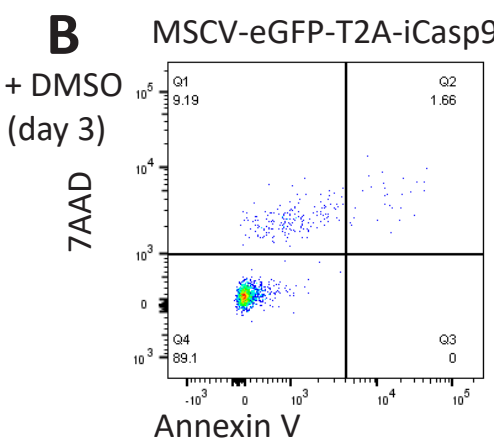

MSCV-eGFP-IRES-iCasp9

MSCV-eGFP-tBID
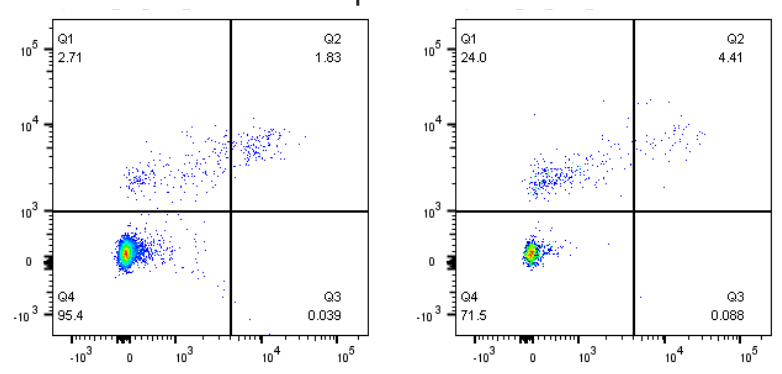

MSCV-eGFP-HSV-TK

+ Inducers: + 10nM AP1903
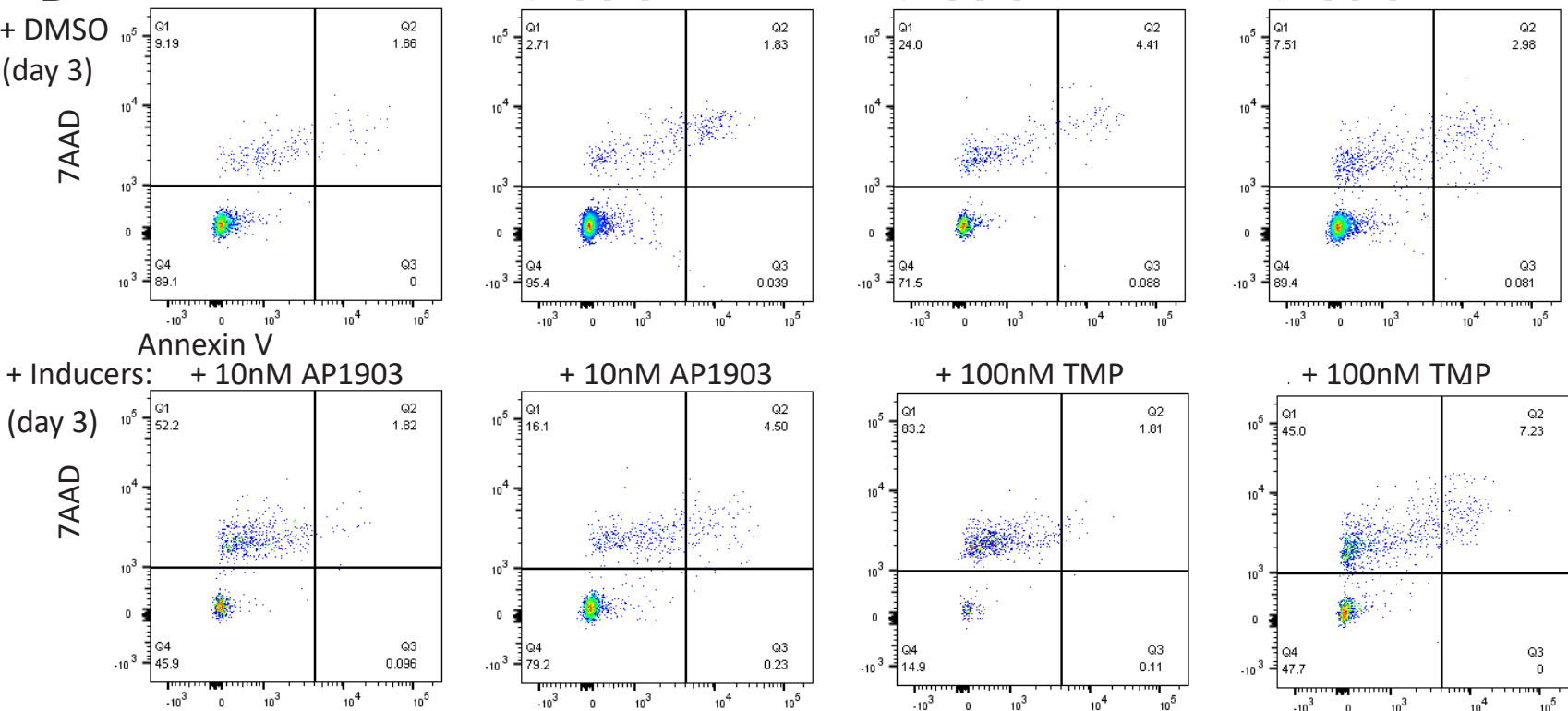

Annexin V

Figure S3 The killing efficacy of NDHFR-tBID safety switch in Jurkat cells. (A) Flow cytometry analysis of Jurkat cells transduced with lentivirus vectors described in Figure $1 A$ on day 1 post treatment. (B) Flow cytometry analysis of Jurkat cells transduced with lentivirus vectors described in Figure $1 \mathrm{~A}$ on day 3 post treatment. 

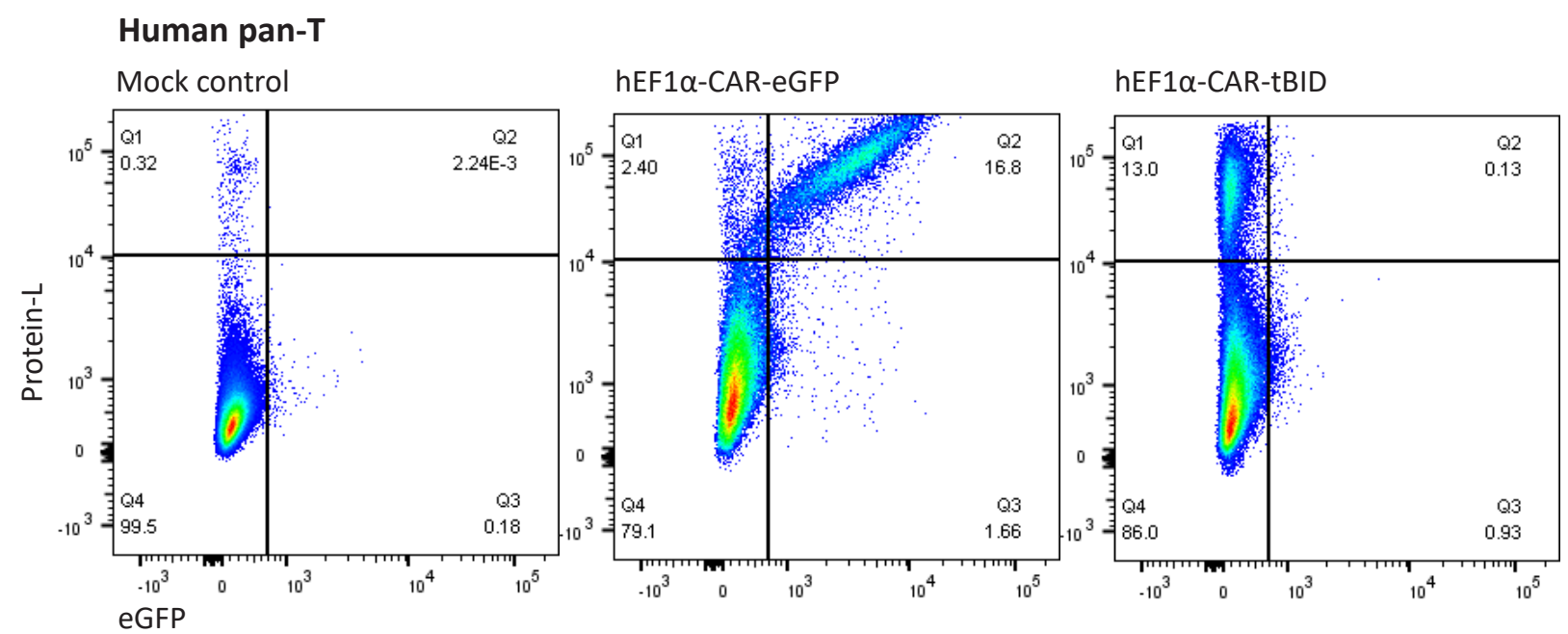

\section{Jurkat}
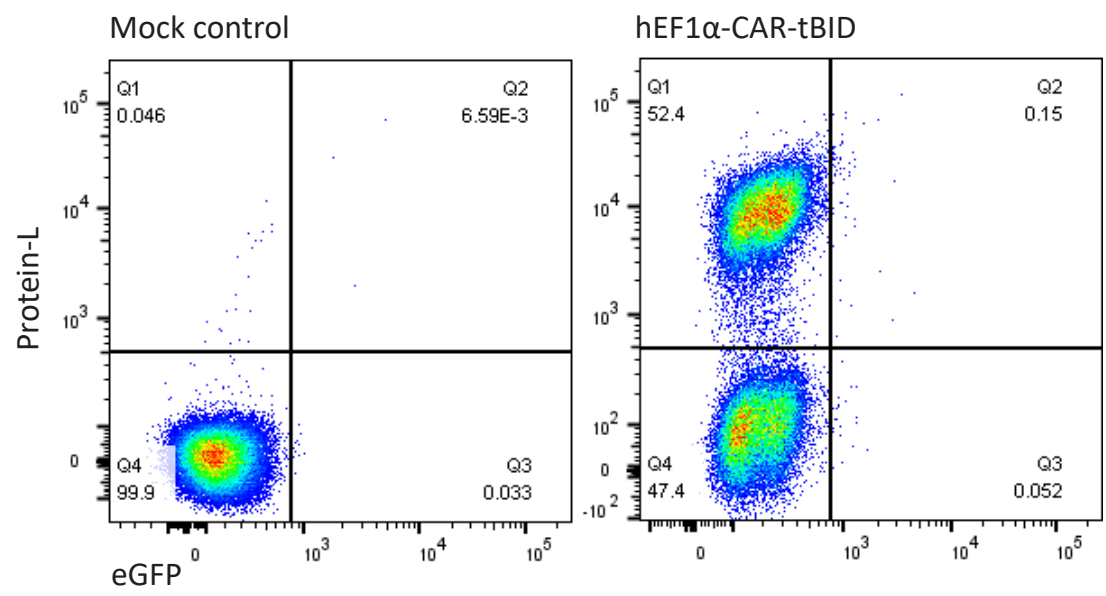

Figure S4 Transduction efficiency of lentiviral vectors in human pan-T cells and Jurkat cells. Lentiviral vectors $b E F 1 \alpha-C A R-e G F P$ and $h E F 1 \alpha-C A R-t B I D$ were transduced into human pan-T cells at $M O I=10$. Lentiviral vector $b E F 1 \alpha-C A R-t B I D$ was transduced into Jurkat cells at MOI $=10$. Both human pan-T cells and Jurkat cells were stained with Annexin V and Protein L followed by flow cytometry analysis on day 3 post transduction. The Annexin V negative single cells were further categorized for eGEP expression and positive Protain L staining. 


\section{Before Protein L Beads Pulldown}

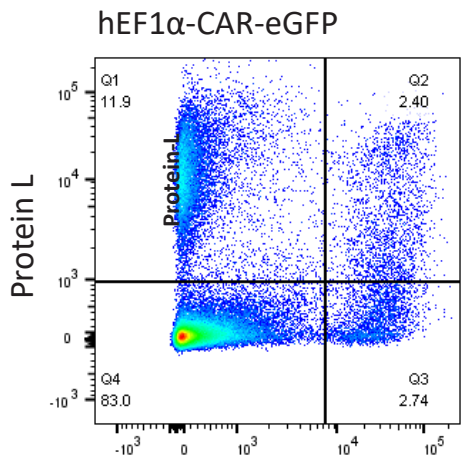

hEF1 $\alpha-C A R-t B I D$

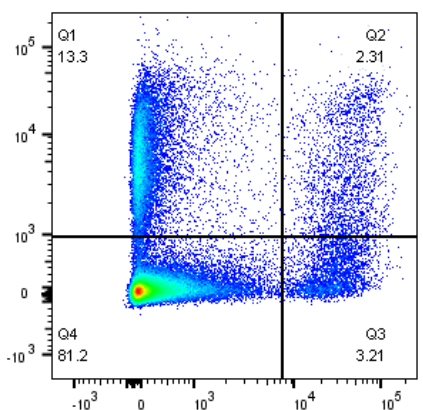

hEF1 $\alpha$-CAR-tBID (M)

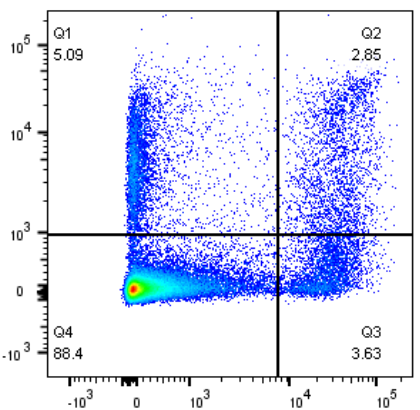

hEF1 $\alpha$-CAR-tBID-CDHFR

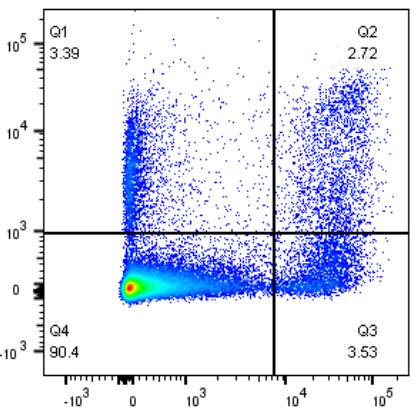

Annexin V

\section{After Protein L Beads Pulldown}

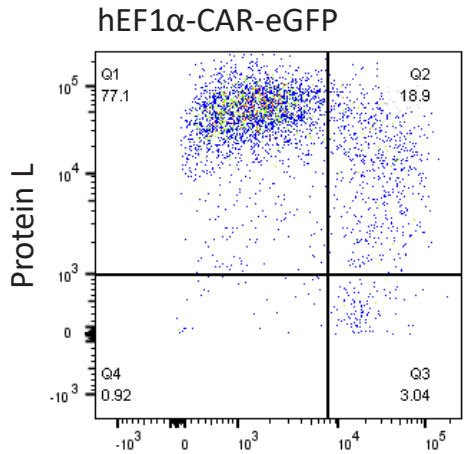

hEF1 $\alpha-C A R-t B I D$

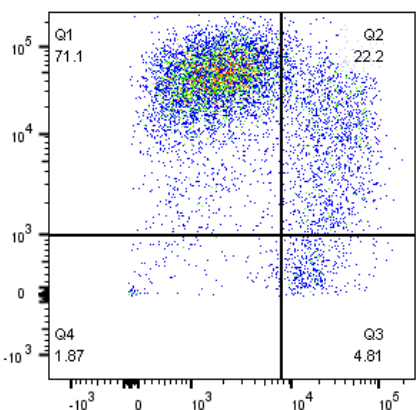

hEF1 $\alpha-C A R-t B I D(M)$

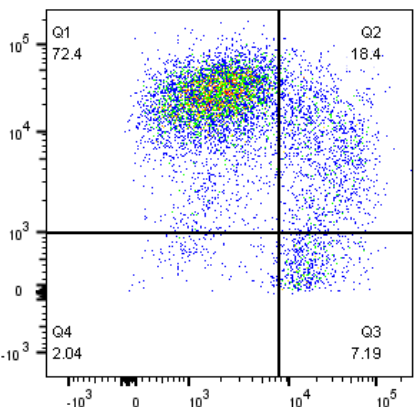

hEF1 $\alpha$-CAR-tBID-CDHFR

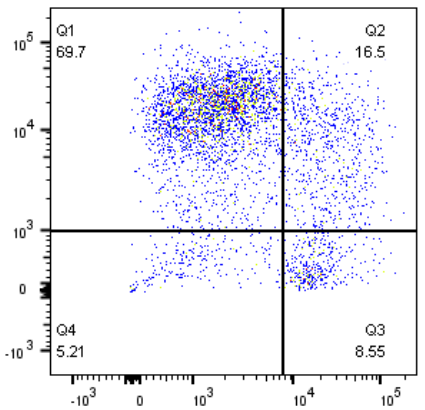

Annexin V

Figure S5 Protein L beads purification of lentiviral vector transduced human pan-T cells.

Table S1 Comparison of HEK293 cell viability during lentivirus packaging

\begin{tabular}{lcc}
\hline Lentiviral Prep Name & In-HouseP24 (VP/mL) & In-House HEK293 Cell Viability (\%) Day of Harvest \\
\hline CAR-NDHFR-tBID & $4.63 E+11$ & 72.0 \\
CAR-iCasp9 & $8.80 E+09$ & 59.7 \\
CAR-eGFP & $5.43 E+11$ & 73.3 \\
\hline
\end{tabular}

